\title{
Exercise interventions to improve postural malalignments in head, neck, and trunk among adolescents, adults, and older people: systematic review of randomized controlled trials
}

\author{
Mohammad Bayattork ${ }^{1,2 *}$, Margrethe Bordado Sköld ${ }^{2,3}$, Emil Sundstrup ${ }^{2}$, Lars Louis Andersen ${ }^{2,4}$ \\ 'Department of Sport Sciences and Physical Education, Faculty of Humanities Science, University of Hormozgan, Bandar Abbas, Iran \\ ${ }^{2}$ National Research Centre for the Working Environment, Copenhagen, Denmark \\ ${ }^{3}$ Department of Occupational and Social Medicine, Holbæk University Hospital, Holbæk, Denmark \\ ${ }^{4}$ Sport Sciences, Department of Health Science and Technology, Aalborg University, Aalborg, Denmark
}

Despite the widespread use of postural correction in exercise interventions, limited experimental evidence exists for its effectiveness. The present study aimed to systematically review the literature on the efficacy of exercise interventions in improving postural malalignment in head, neck, and trunk. A systematic review was performed by screening four scientific databases (MEDLINE, Web of Science, EBSCO, and Cochrane database) for published randomized controlled trials (RCTs) in English from 1996-2019. The review followed the PRISMA (Preferred Reporting Items for Systematic Reviews and Meta-Analyses) statement guidelines. Two researchers independently performed study screening, extracting data and assessing the risk of bias for each included study using the Cochrane Collaboration tool for evaluating the risk of bias. A total of 22 RCTs comprising 1,209 participants were identified for in- clusion in the review. There was a high risk of bias across most of the included studies (12 studies). Only two studies were classified as low risk of bias, and eight studies were classified as moderate risk of bias. The intervention duration ranged from 2 to 13 weeks, frequency from 2 to 4 days per week, and duration of each session between 15 to $60 \mathrm{~min}$. The insufficiency and quality of included studies did not allow an integrated assessment of the efficacy of exercise interventions on postural malalignments; however, the positive effects noticed in most of the studies indicate some advantages but underscores the necessity of adequately designed RCTs in this field.

Keywords: Exercise, Posture, Malalignment, Adolescents, Adults

\section{INTRODUCTION}

Postural malalignment refers to a considerable displacement or deviations from the normal alignment in a kinetic chain resulting in abnormal biomechanical stress that can compromise the ability to deal with these forces (Schamberger, 2016). The occurrence of postural deviations is high in school-aged children and adolescents; while some changes are common in human growth and development, others may harm the quality of life (Schwanke et al., 2016). Besides, malalignment may occur among adults or during the ag- ing process and may damage the pulmonary function and the quality of life (Bansal et al., 2014). Also, major deviations from optimal alignment can be aesthetically unpleasant for people, impact their efficiency of muscles, and predispose them to neurological or musculoskeletal disorders (Hrysomallis and Goodman, 2001).

Clinicians believe that it is essential to quantify head, shoulder, and spinal posture behavior because they influence and are influenced by many biomechanical, motor control and performance variables (Claus et al., 2016). In this regard, it has been assumed that exercise can correct the postural malalignment. Interestingly,

\footnotetext{
${ }^{*}$ Corresponding author: Mohammad Bayattork

(iD) https://orcid.org/0000-0002-7588-0079

Department of Sport Sciences and Physical Education, Faculty of Humanities

Science, University of Hormozgan, Bandar Abbas 7916193145, Iran

E-mail: Mohammadbayat82@gmail.com

Received: January 17, 2020 / Accepted: February 5, 2020
} 
despite the widespread inclusion of postural correction in exercise interventions, there is limited or contradictory experimental data to support its effectiveness, and little is known regarding which exercise is the most efficient intervention (Harman et al., 2005). Two previous review found little evidence to support this assumption due to insufficiency of reliable and valid data gathered in randomized controlled settings to help the dispute that exercise intervention will improve the postural malalignments (Bansal et al., 2014; Hrysomallis and Goodman, 2001). By contrast, two recent systematic reviews and meta-analysis found the effectiveness of exercise for improving postural malalignments (González-Gálvez et al., 2019; Sheikhhoseini et al., 2018). However, one suggested that the therapeutic exercises may result in changes in head posture deviation, but the precise nature of its improvement remains to be established (Sheikhhoseini et al., 2018). Therefore, it seems evidence-based recommendations for practitioners about effective exercises for individuals with postural malalignments are needed. Also, knowledge of feasible and efficient exercise intervention strategies with describing precise details to improving postural malalignments or prevent its consequences are, therefore, warranted by patients, practitioners, and rehabilitation centers.

Previous systematic reviews differ from our review in many aspects. Most of them had a narrower scope (Hrysomallis and Goodman, 2001) and/or were restricted to specific malalignments and participants (e.g., adolescents with scoliosis) (Romano et al., 2013) or older people with hyperkyphosis (Bansal et al., 2014). Also, there are some differences in the search strategy and inclusion criteria of our systematic review compared with theirs (González-Gálvez et al., 2019).

The current systematic review will focus on the effect of exercise interventions on different types of malalignments in adolescents, adults and older people and will, therefore, embrace a broader area than other systematic reviews.

This systematic review of RCTs aimed to evaluate the effectiveness of exercise interventions on improving postural malalignment in the head, shoulder, scapular, and spine among adolescent, middle-aged and older people. Subsequently, the key issues impacting on the outcome, including intervention type, volume, duration, and dose, will be identified.

\section{MATERIALS AND METHODS}

\section{Protocol and registration}

The protocol of this systematic review was registered in the international prospective register of systematic reviews (PROSPE-
RO) site on 07/06/2018 (CRD42018096531).

\section{Eligibility criteria for considering studies for this review}

This systematic review was designed collectively by the members of the review team following the PRISMA (Preferred Reporting Items for Systematic Reviews and Meta-Analyses) guidelines (Shamseer et al., 2015). Studies were selected according to the criteria outlined below.

\section{Study designs}

We included only RCTs, and nonrandomized controlled trials were excluded.

\section{Types of participants}

We included studies in which all patients were diagnosed as having one or a combination of types of postural malalignments in their head, shoulder, scapular, and spine. In our protocol, we initially mentioned the scoliosis angle as one of our primary outcomes. After performing the initial study searches; however, we chose to remove this criterion due to the abundance of recently systematic reviews in which investigated the effectiveness of exercise intervention on the improvement of scoliosis. Also, we excluded studies in which patients had a specific condition or problem such as pregnancy, osteoporosis, spondylosis, and any neurological diseases, e.g., Parkinson. No restrictions regarding gender were made.

\section{Types of interventions}

All types of exercise interventions (such as strengthening, stretching, aquatic, yoga, and Pilates exercise or any other exercise with a focus on treatment or prevention of postural malalignment) were included as long as the participants performed at least a part of the exercises independently. Sport or active recreational activity was not considered as an exercise intervention for improving postural malalignments, and studies including these types of activity or active exercise interventions without any reported outcome measures for improving postural malalignment were excluded. No restrictions regarding clinical setting (in-patients or out-patients) were made.

\section{Types of outcome}

The primary outcome was the interventional effect on degrees of postural malalignments (forward head, round shoulder, thoracic kyphosis, lumbar lordosis, and scapular position) as measured by the observational or clinical methods. Studies were not excluded 
based on the type of measurement used to assess postural malalignments. Secondary outcomes were the key issues impacting on the primary outcome, including intervention type, volume, duration, and dose.

\section{Language and time frame}

We included only articles written in the English language and published within the last 23 years were included.

\section{Information sources and search strategy}

Literature search strategies were developed using medical subject headings and text words related to exercise interventions AND postural malalignments within the last 23 years. The following databases were searched: Cochrane, Web of Science, MEDLINE, and EBSCO databases. These databases were chosen since they include many RCTs in the health field, including the leading journals publishing trials with exercise interventions. The search strategies used in databases are displayed in Supplementary material. A hand search of reference lists was also performed. The last search was run on April 20, 2019.

\section{Study records}

Literature search results were uploaded to EndNote Software, which facilitated collaboration among reviewers during the study selection process and was used for sorting and duplicate removal. The review authors (MB and MBS) independently screened the titles and abstracts yielded by the search against the inclusion criteria. Review author pairs then screened the full-text reports and decided whether these met the inclusion criteria. Any disagreement between the review authors was resolved by a third review-team member (ES).

\section{Data collection process}

Data extraction forms were developed based on the Cochrane Back Review Group (Van Tulder et al., 2003). The data extraction form was pilot-tested on four studies, and refined accordingly. The reviewers extracted data independently and in duplicate from each eligible study. Also, reviewers resolved any disagreements by discussion or by a third person if needed.

\section{Risk of bias assessment from individual studies}

The reviewer authors assessed the risk of bias assessment for all included studies. The information was collected using the Cochrane Collaboration tool for assessing the risk of bias (Table 8.5.a in the Cochrane Handbook for Systematic Reviews of Interven- tions), that includes: selection bias, performance bias, detection bias, attrition bias, reporting bias, and other sources of bias (Higgins et al., 2011). A judgment as to the possible risk of bias on each of the six domains was made from the extracted information, rated as 'high risk,' 'low risk,' or 'unclear.' Then, each included study is assigned an overall grade of low, moderate or high risk of bias based on the evaluation of the six domains and according to the following criteria: low risk of bias if the first three domains are noted as 'yes,' and no serious concerns regarding the last three domains were identified; moderate risk of bias if one or two domains are noted as unclear; and high risk of bias if more than two domains are noted as unclear (Higgins et al., 2011). Two review authors made these judgments independently and any disagreement was discussed. A third review author consulted if disagreements persisted.

\section{Analysis and GRADE approach}

Our systematic review included a variety of exercise interventions and participants (people with different kind of postural malalignments). Therefore, the gathered data is prone to high heterogeneity, which discourages a meta-analysis, and therefore, only a qualitative synthesis was considered in this review. The quality of evidence for all outcomes was rated based on the GRADE (Grading of Recommendations, Assessment, Development and Evaluations)

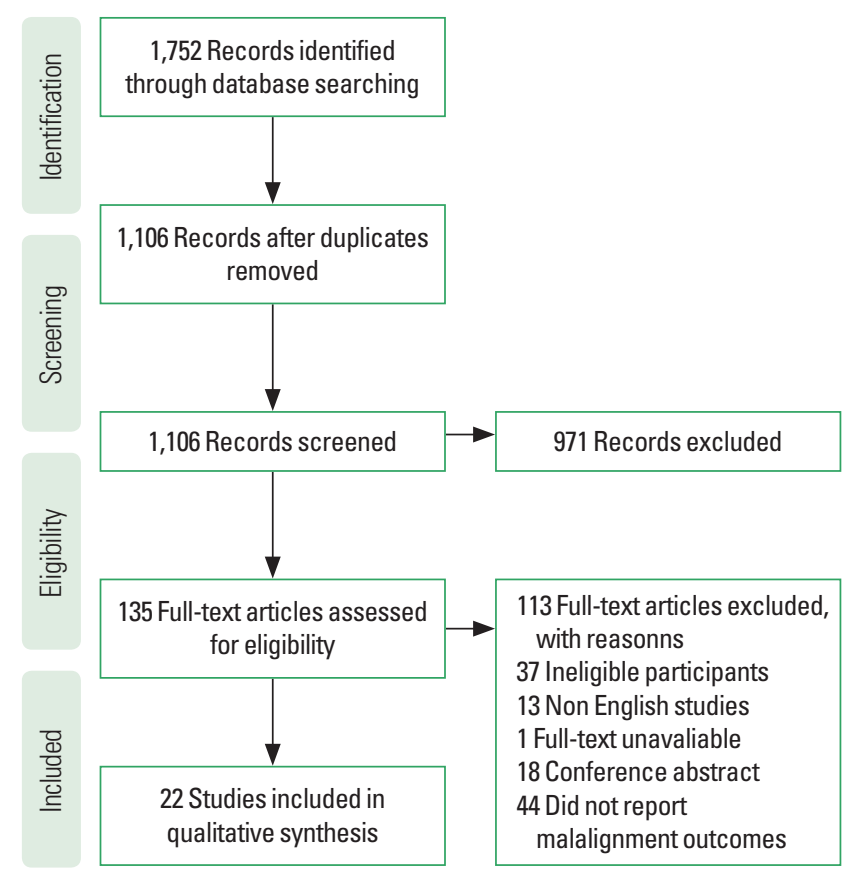

Fig. 1. Flow diagram. 


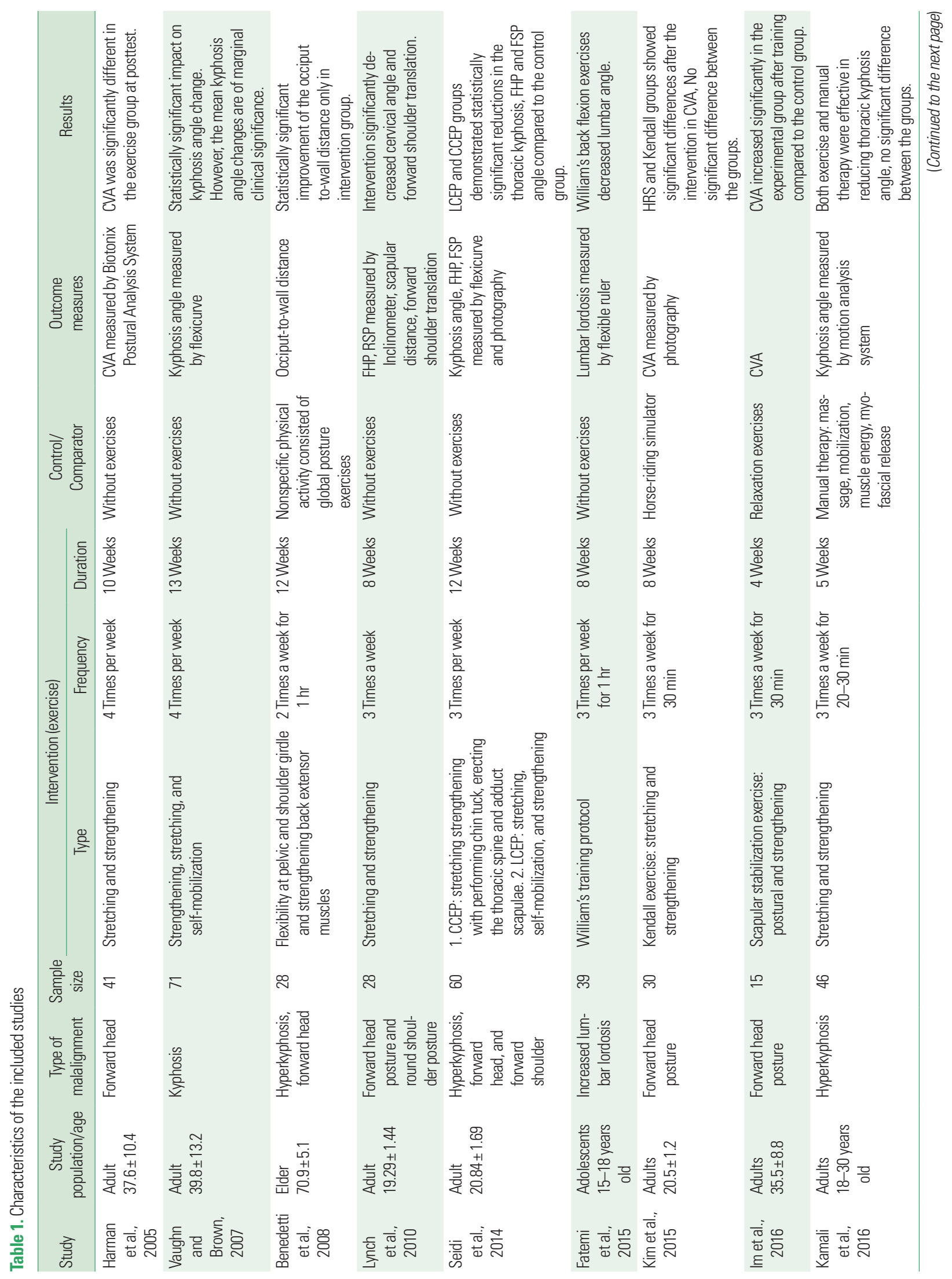




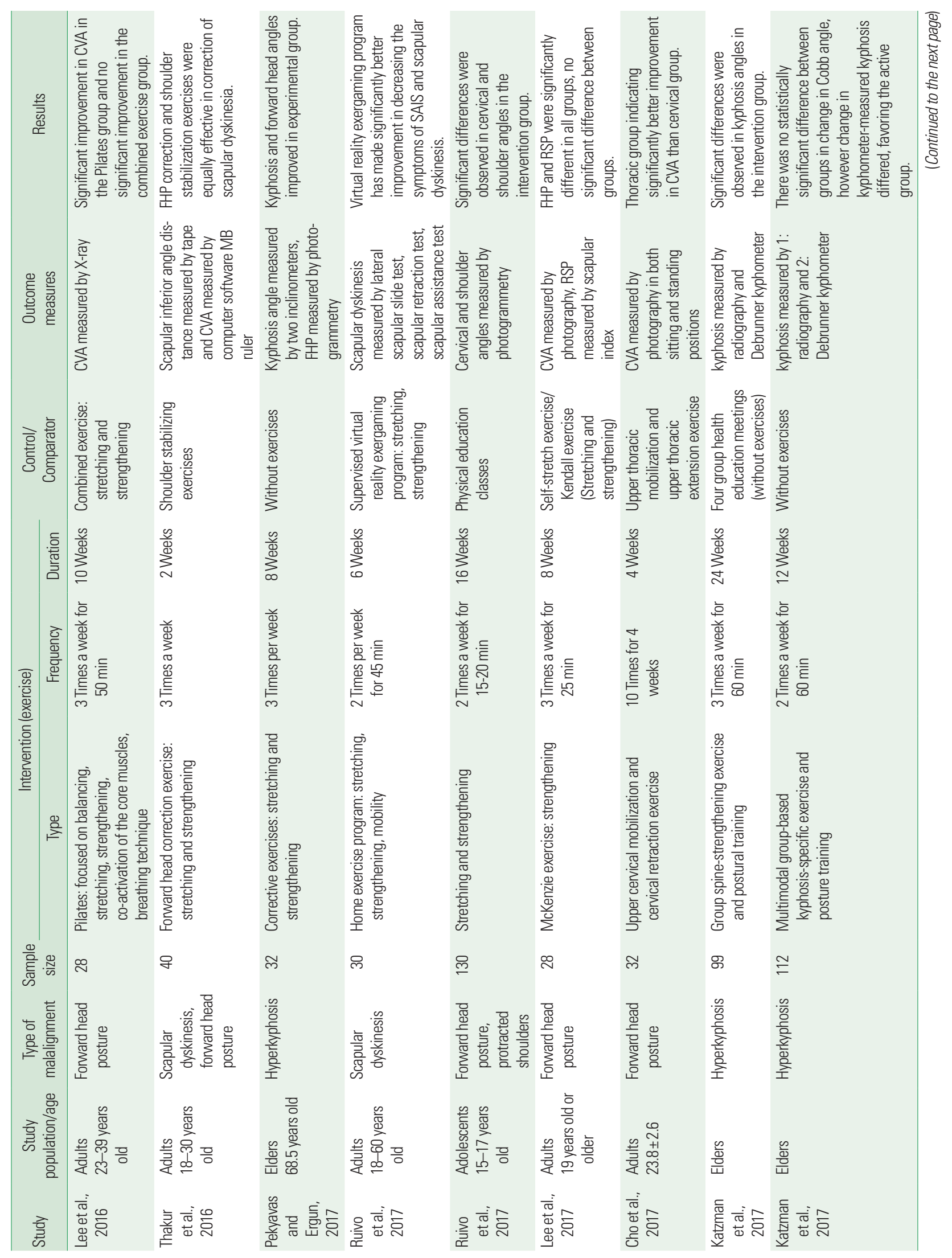




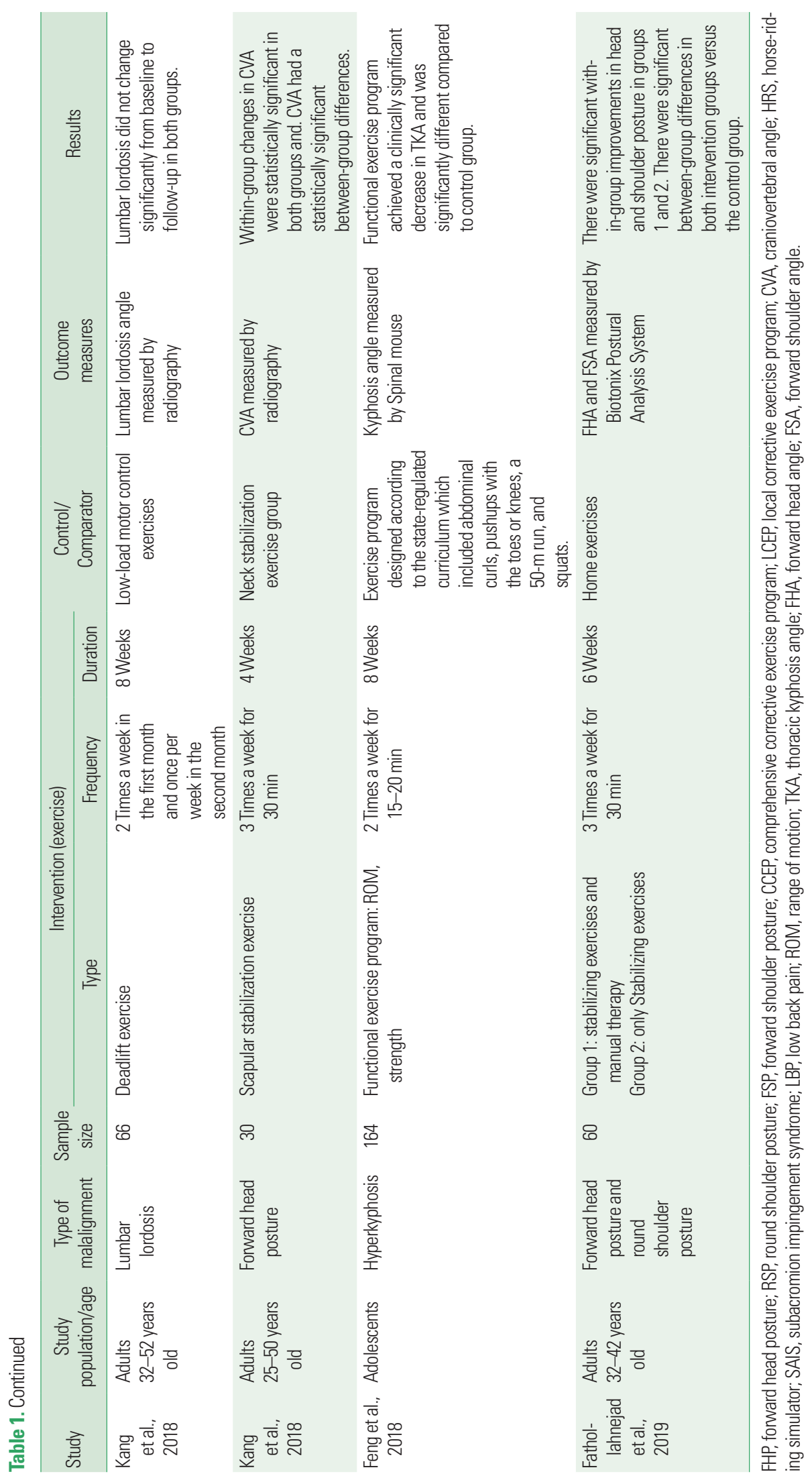


approach (Balshem et al., 2011). The quality rating of the evidence started at high. It was rated down to moderate, low or very low evidence according to the following guidelines: (a) risk of bias this rated down if more than $25 \%$ of the subjects were from studies with a high risk of bias; (b) inconsistency of results - this was downgraded if significant differences in magnitude and direction of effects between included studies was present; (c) indirectness this rated down if more than $50 \%$ of the subjects were outside the target group; and (d) imprecision- following Rubinstein et al. (2013), single studies with $<400$ subjects for continuous outcomes or $<300$ subjects for dichotomous outcomes were considered as 'low-quality evidence,' and 'very low-quality evidence' if there were further limitations (of high risk of bias, inconsistency, or indirectness) on the GRADE approach.

\section{RESULTS}

\section{Study selection and characteristics}

A total of 22 RCTs were identified for inclusion in the review. The flow diagram in Fig. 1 illustrates the selection pro- cess and characteristics of the 22 included studies can be seen in Table 1.

\section{Risk of bias and quality of evidence within studies}

The results of assessing the risk of bias within studies are shown in Table 2. To determinate the interrater reliability for authors, Cohen Kappa was calculated $(\mathrm{Kappa}=0.891)$ and found a high level of agreement (McHugh, 2012). Moreover, Tables 3 shows the synthesized results based on the GRADE approach described above. Overall, there was very low- to moderate-quality evidence to suggest that exercise interventions can improve postural malalignments in adolescents, adults, and older people.

\section{DISCUSSION}

The current systematic review of RCTs was conducted to investigate the effects of exercise interventions on improving postural malalignments among adolescents, adults, and older people. Secondarily, to explore the influence of intervention type, volume, duration, and the dose of exercise. Our systematic review showed

Table 2. Results of assessing risk of bias within studies

\begin{tabular}{|c|c|c|c|c|c|c|c|c|}
\hline Study & $\begin{array}{l}\text { Random } \\
\text { sequence } \\
\text { generation }\end{array}$ & $\begin{array}{c}\text { Allocation } \\
\text { concealment }\end{array}$ & $\begin{array}{c}\text { Blinding of } \\
\text { participants and } \\
\text { personnel }\end{array}$ & $\begin{array}{l}\text { Blinding of } \\
\text { outcome } \\
\text { assessment }\end{array}$ & $\begin{array}{c}\text { Incomplete } \\
\text { outcome data }\end{array}$ & $\begin{array}{l}\text { Selective } \\
\text { reporting }\end{array}$ & $\begin{array}{c}\text { Other sources of } \\
\text { bias }\end{array}$ & $\begin{array}{l}\text { Overal grade of } \\
\text { risk of bias }\end{array}$ \\
\hline Harman et al., 2005 & Yes & Unclear & Unclear & Yes & Yes & Yes & Yes & Modrate \\
\hline Vaughn and Brown, 2007 & Yes & Unclear & Yes & Yes & Yes & Yes & Yes & Modrate \\
\hline Benedetti et al., 2008 & Unclear & Unclear & Unclear & Yes & Yes & Yes & Yes & High \\
\hline Lynch et al., 2010 & Yes & Unclear & No & No & Yes & Yes & Yes & High \\
\hline Seidi et al., 2014 & Unclear & Unclear & Unclear & Unclear & Yes & Yes & Yes & High \\
\hline Fatemi et al., 2015 & Unclear & Unclear & Unclear & Unclear & Yes & Yes & Yes & High \\
\hline Kim et al., 2015 & Unclear & Unclear & Unclear & Unclear & Yes & Yes & Yes & High \\
\hline Im et al., 2016 & Unclear & Unclear & Unclear & Unclear & Unclear & Yes & Unclear & High \\
\hline Kamali et al., 2016 & Yes & Unclear & Yes & Yes & Yes & Yes & Yes & Modrate \\
\hline Lee et al., 2016 & Unclear & Unclear & Yes & Unclear & Yes & Yes & Yes & High \\
\hline Thakur et al., 2016 & Unclear & Unclear & No & Unclear & Yes & Yes & Yes & High \\
\hline Bahrekazemi et al., 2017 & Unclear & Unclear & Unclear & No & Yes & Yes & Yes & High \\
\hline Pekyavas and Ergun, 2017 & Yes & Unclear & Unclear & Unclear & Yes & Yes & Yes & High \\
\hline Ruivo et al., 2017 & Yes & Yes & Yes & Yes & Yes & Yes & Yes & Low \\
\hline Lee et al., 2017 & Unclear & Unclear & Unclear & Unclear & Yes & No & Unclear & High \\
\hline Cho et al., 2017 & Yes & Unclear & Yes & Yes & Yes & Yes & Yes & Modrate \\
\hline Katzman et al., 2017 & Yes & Yes & Unclear & Yes & Yes & Yes & Yes & Modrate \\
\hline Katzman et al., 2017 & Yes & Unclear & Unclear & Unclear & Yes & Yes & Yes & Modrate \\
\hline Berglund et al., 2018 & Yes & Unclear & Yes & Yes & Yes & Yes & No & Modrate \\
\hline Kang et al., 2018 & Unclear & Unclear & Unclear & Unclear & Yes & Yes & Yes & High \\
\hline Feng et al., 2018 & Yes & Unclear & Yes & Unclear & Yes & Yes & Yes & Modrate \\
\hline Fathollahnejad et al., 2019 & Yes & Yes & Yes & Yes & Yes & Yes & Yes & Low \\
\hline
\end{tabular}


Table 3. GRADE (Grading of Recommendations, Assessment, Development and Evaluations) evidence profile: exercise intervention for people with postural malalignments

\begin{tabular}{|c|c|c|c|c|c|c|}
\hline \multirow[b]{2}{*}{ Outcome (No. of studies) } & \multicolumn{5}{|c|}{ Quality assessment } & \multirow{2}{*}{$\begin{array}{l}\text { Summary of } \\
\text { findings Quality }\end{array}$} \\
\hline & $\begin{array}{l}\text { Limitations of study } \\
\text { design }\end{array}$ & Inconsistency & Indirectness & Imprecision & Publication bias & \\
\hline All types of malalginments & Serious & Serious & No serious & No serious & Undetected & $\bigoplus \underset{\text { Low }}{\oplus \bigcirc \bigcirc}$ \\
\hline Forward head posture (13) & Serious & No serious & No serious & No serious & Undetected & $\underset{\text { Moderate }}{\bigoplus \oplus \bigoplus \bigcirc}$ \\
\hline Forward/round shoulder posture (4) & Serious & No serious & Unclear & Serious & Undetected & $\underset{\text { Low }}{\oplus \bigcirc \bigcirc}$ \\
\hline Scapular dyskinesis (2) & Serious & No serious & Unclear & Serious & Undetected & $\underset{\text { Low }}{\oplus \bigcirc \bigcirc}$ \\
\hline Hyperkyphosis (8) & No serious & Serious & No serious & No serious & Undetected & $\underset{\text { Moderate }}{\bigoplus \oplus \bigoplus \bigcirc}$ \\
\hline Hyperlordosis (2) & Serious & Serious & Unclear & Serious & Undetected & $\underset{\text { Very low }}{\oplus \bigcirc \bigcirc}$ \\
\hline
\end{tabular}

that the available evidence on the efficacy of exercise in postural malalignments is insufficient and mainly of low or moderate quality. However, 21 included studies showed a significant improvement after conducting the exercise intervention, and only one study did not show a significant improvement (Berglund et al., 2018). Also, no evidence was identified that the exercises negatively or harmfully affect postural malalignments. Although we included RCTs in order to assess only high-quality studies, there was a moderate or high risk of bias and poor reporting across most of the studies.

It has been postulated that if some segments of the body are maintained out of the optimal posture for a long time, the muscles may remain in shortened or lengthened positions. These positions may result in adaptive lengthening or shortening over time (Hrysomallis and Goodman, 2001). Therefore, it has been assumed that corrections in the poor posture would be reached with improved muscle strength and length or other factors related to malalignments, such as related movement patterns and improved postural awareness. While a causal association may not necessarily exist between this postulation and hypothesis, all reviewed exercise interventions focused, in part, on improving malalignments by stretching the shortened muscles and strengthening the weakened muscles in the involved area based on the traditional approach (Sahrmann et al., 2017). Few included RCTs in this review evaluated the effectiveness of the exercise interventions on muscle weakness or shortness (Benedetti et al., 2008; Fatemi et al., 2015); therefore, it is unclear whether participants had related weak or short muscles at baseline or if the intervention had a significant effect on muscle weakness or shortness, or whether such a phenomenon as 'shortened muscles' due to certain exposures is real. In fact, studies using stretching exercise shows no change in muscle length, but rather improved tolerance to stretching (Magnusson, 1998; Magnusson et al., 1997). Besides, the researchers employing the neurological approach generally believe that the basis of the functional approach in musculoskeletal problems is the interaction of all central and peripheral nervous systems, muscular and skeletal structures in the production and control of motion (Hamill et al., 1999; Morris et al., 2006). Further, they believe that it is essential considering to the individual's aspects of posture, muscle activation, and movement pattern (Hodges et al., 2019). While none of the RCTs reviewed assessed the muscle activation and related movement patterns as an initial strategy to provides information for prescribing the exercise intervention.

\section{Comparison with other systematic reviews}

Three systematic reviews and a narrative review were identified in which it has been examined a comparable research question (Bansal et al., 2014; González-Gálvez et al., 2019; Hrysomallis and Goodman, 2001; Sheikhhoseini et al., 2018). Although one would hope that multiple systematic reviews would make higher clarification on the effects of exercise interventions on improving postural malalignments, we also discovered some discrepancies. The explanations for the discordances from these systematic reviews in comparison with our systematic review are regarding the methodology.

In a recent systematic review, Sheikhhoseini et al. (2018) have examined the effectiveness of exercise interventions on improving forward head posture. His review suggested there is strong evi- 
dence showing an improvement in forward head posture in response to exercise intervention. Therefore, there were some differences in the final messages explained by: (a) the restriction to only forward head posture in Sheikhhoseini et al. (2018) whereas we used broader inclusion criteria of malalignments, (b) the current review was restrict to malalignment outcomes whereas Sheikhhoseini et al. (2018) added neck pain outcomes in addition to forward head angle, (c) in our review we excluded studies in which patients had a specific problem whereas Sheikhhoseini et al. (2018) included participants with cervical spondylotic radiculopathy and myofascial pain, (d) additional articles published since the review of Sheikhhoseini et al. (2018) was conducted, (e) progress of quality evaluation criteria that resulted in several contrasts in quality assessment scoring.

Two previous reviews have been studied the effectiveness of exercise intervention on improving hyperkyphosis malalignment (Bansal et al., 2014; Hrysomallis and Goodman, 2001). A recent systematic review by Bansal et al. (2014) summarized that the insufficiency and quality of included studies did not allow an integrated assessment of the efficacy of exercise interventions on improving thoracic kyphosis angle. However, due to the presence of positive effects in high-quality studies, it seems that there are some advantages, and they indicate the necessity of adequately designed RCTs examining the effectiveness of exercise intervention on hyperkyphosis (Bansal et al., 2014). Despite that both our review and Bansal et al. (2014) have a similar conclusion, there were some differences related to population and study design. Our review included studies in which participants were adolescents, adults and older people without specific diseases. In contrast, in Bansal review, the inclusion of individuals aged $\geq 45$ was used to review studies of postmenopausal women with osteoporosis. Also, we only included RCTs whereas the Bansal et al. (2014) included both RCTs and nonrandomized intervention trials.

The other review identified, Hrysomallis and Goodman (2001), was not a systematic review. Here, the authors conducted a review of improving postural malalignment and resistance exercise (Hrysomallis and Goodman, 2001). They found the insufficiency of reliable and valid data gathered in randomized controlled settings to help the dispute that exercise intervention will improve the postural malalignments such as hyperkyphosis. The same, objective data to demonstrate that exercise intervention will result in postural malalignments was not available. Similarly, our review found considerable evidence about the effectiveness of exercise intervention on postural malalignments such as hyperkyphosis and hyperlordosis to be scarce and mainly of low or moderate quality.
Another recent systematic review by González-Gálvez et al. (2019) summarized there were significant effects of the exercise intervention on decreasing the angle of thoracic kyphosis, although there were no considerable effects on the lumbar lordosis angle (González-Gálvez et al., 2019). Despite their review compared with ours had the same number of included studies and considerable overlap between them with four of 10 studies (40\%) regarding the kyphosis and lordosis malalignments, there were still some differences in the conclusion. Our different inclusion criteria can explain some of these differences. The review of González-Gálvez et al. (2019) only examined the effect of exercise programs on the angle of kyphosis and lordosis, not the improvement of hyperkyphosis and/or hyperlordosis angle in people with these malalignments. Most of the participants of included studies in a review of González-Gálvez et al. (2019) did not have the hyperkyphosis or hyperlordosis malalignments (Ko et al., 2018; Muyor et al., 2012). It seems that they have been included only because of the kyphosis and lordosis angles was measured. In addition, although he included only RCTs, the study of Jang et al. (2019) was not an RCT.

Regarding scapular dyskinesis, despite many observational and experimental studies and some review articles in this field (Cools et al., 2007; Cools et al., 2014), to best of our knowledge, a systematic review to investigate the effect of exercise on improving scapular dyskinesis has not previously been performed. Therefore, this current review remains the only review and we could not compare our results with others.

\section{The key issues impacting on the outcome}

For another aim of the current review, the studies reviewed will be discussed regarding the undertaken frequency of exercises, the performed intensity of exercises, the duration of exercises, and the type of exercises.

The frequency of exercises differed in reviewed studies (Table 1) with exercise protocol ranging from 2 to 4 sessions weekly with profits visible from all frequencies. Many of the included studies demonstrating significant improvement in the postural malalignment, used the frequency of 2 to 4 sessions per week. Two studies with high quality applied 2 and 3 sessions per week (Fathollahnejad et al., 2019; Ruivo et al., 2017). It seems that exercise protocols of at least 2 to 3 times a week presented gains in neuromuscular adaptations, which is in accordance with recognized exercise advantages and its efficiency as accepted by the American College of Sports Medicine guidelines (Garber et al., 2011). Moreover, the frequency of exercise was defined per the study design (e.g., 2 sessions/wk.), but the adherence to the intervention found to differ 
considerably and previous studies, therefore, looked at issues related to exercise compliance. Harman et al. (2005) demonstrated a 91\% compliance rate with exercises intervention frequency of 4 sessions over 10 weeks. However, an exercise protocol with high frequency maybe not assume suitable for participants with postural malalignments due to the barriers of adherence. Therefore, findings suggest that the most effective exercise frequency to aim improving postural malalignments is ranging from 2 to 4 sessions with an average of 3 times a week.

It seems to be difficult determining the real-time spent for exercises throughout intervention because there was a lack of adherence by subjects when a supervised training protocol was entirely done. The duration of the exercise session was between 15 to 60 $\min$ in the reviewed studies; whereas, exercise protocols were between 2 to 24 weeks (Table 1). Many studies have shown that at least 4 to 8 weeks' resistance training is the best time to obtain positive exercise characteristics such as strength, endurance, and neuromuscular changes (Eklund et al., 2015). Most of the included studies in our systematic review also were in this range. However, there were two studies with a short duration (2 weeks) (Thakur et al., 2016) or a long duration (24 weeks) (Katzman et al., 2017). When formulating an optimally effective intervention, the essential thing that must be considered is that how likely an individual is to adhere entirely to a long duration program, or the short duration may be insufficient in inducing significant and clinical effects. Therefore, when assessing the spending time of an exercise and the whole duration of a training intervention to produce the best results, it is suggested that exercise protocol should last at least 6 weeks for occurring the neuro-physiological advantages. Exercising between 15 and 60 min provides the most optimal results with 30 to 45 min being a reasonable and mainly achievable exercise session duration.

Regarding the type of exercise, although there has been much argument as to which type of exercise is most effective, a combination of both stretching and strengthening obtains the most considerable advantages for subjects. The studies demonstrated immediate and some long-term advantages in improving postural malalignments resulting in increased muscle length or strength, because it is widely postulated that some muscles are substantially weaker or shorter in those with postural malalignments (Sahrmann et al., 2017). However, the researchers using a neurological approach believe that changes subsequent to the postural malalignments are based on the interaction of the central and peripheral nervous systems, as well as the muscular and skeletal structures (Hamill et al., 1999; Morris et al., 2006). In this functional ap- proach, the musculoskeletal problems are thought to be due to the role of muscles in motor function; furthermore, changes in the alignment are not only a change in the muscle length and strength, but represent more important changes in muscle neuromuscular factors, such as muscle recruitments (Janda, 1983). Therefore, it seems that the best result would occur when an applied strengthening/stretching intervention can focus on both the neural as well as muscular components of the malalignment.

\section{Future research priorities}

While there is a lack of high-quality study in this field, those reviewed in the current study would demonstrate that it is a field of research worth pursuing. It seems to be advantageous to investigate the effectiveness of exercise interventions in improving postural malalignments, especially with a long period of intervention and follow-up measurements. Future RCTs should be more precise on detailed allocation concealment and blinding. Moreover, they should report effect size for the primary interventional outcome in addition to statistically significant differences. Also, it would be useful to study the effectiveness of exercise interventions in improving postural malalignments with assessment methods related to muscle activations and movement patterns in addition to alignments before and after an exercise intervention. While it may be challenging to recruit a large number of people for each study, many high-quality RCTs can be pooled subsequently in a meta-analysis.

\section{Limitations}

This is the first systematic review that included studies with five different postural malalignments based on a broad search design. However, it may also consider as a limitation so that, due to the heterogeneity in terms of interventions and outcome measurements of the reviewed studies, performing a meta-analysis was not meaningful. The inclusion of only English written literature, which might have led to the exclusion of important findings and the exclusion of the gray literature were the limitations of the current study. However, RCTs are seldom published only in the gray literature. Also, due to time restrictions, the review authors could not request specific information about the studies from the study authors.

\section{CONCLUSIONS}

The majority of the RCTs published within the last 23 years found some positive effects of exercise interventions for postural 
malalignments. However, most of the studies were rated poorly in terms of quality assessment when using the Cochrane Collaboration tool for assessing the risk of bias and GRADE approach for quality of evidence. This supports the need for an adequately designed high-quality RCTs examining the effect of exercise on postural malalignments. Moreover, the best result for improving postural malalignments would occur when an applied strengthening/ stretching intervention can focus on both the neural and muscular components and last at least 6 weeks with an average of 3 times a week, between 15 and $60 \mathrm{~min}$.

\section{CONFLICT OF INTEREST}

No potential conflict of interest relevant to this article was reported.

\section{SUPPLEMENTARY MATERIAL}

Supplementary material can be found via https://doi.org/10. 12965/jer.2040034.017.

\section{REFERENCES}

Bahrekazemi B, Letafatkar A, Hadadnezhad M. The effect of eight weeks of global postural corrective exercises on kyphosis and forward head angle in elderly women with age-related hyperkyphosis. Int J Med Res Health Sci 2017;6:40-44.

Balshem H, Helfand M, Schünemann HJ, Oxman AD, Kunz R, Brozek J, Vist GE, Falck-Ytter Y, Meerpohl J, Norris S, Guyatt GH. GRADE guidelines: 3. Rating the quality of evidence. J Clin Epidemiol 2011;64: 401-406.

Bansal S, Katzman WB, Giangregorio LM. Exercise for improving age-related hyperkyphotic posture: a systematic review. Arch Phys Med Rehabil 2014;95:129-140.

Benedetti MG, Berti L, Presti C, Frizziero A, Giannini S. Effects of an adapted physical activity program in a group of elderly subjects with flexed posture: clinical and instrumental assessment. J Neuroeng Rehabil 2008;5:32.

Berglund L, Aasa B, Michaelson P, Aasa U. Sagittal lumbopelvic alignment in patients with low back pain and the effects of a high-load lifting exercise and individualized low-load motor control exercises-a randomized controlled trial. Spine J 2018;18:399-406.

Cho J, Lee E, Lee S. Upper thoracic spine mobilization and mobility exercise versus upper cervical spine mobilization and stabilization exercise in individuals with forward head posture: a randomized clinical trial.
BMC Musculoskelet Disord 2017;18:525-535.

Claus AP, Hides JA, Moseley GL, Hodges PW. Thoracic and lumbar posture behaviour in sitting tasks and standing: progressing the biomechanics from observations to measurements. Appl Ergon 2016;53 Pt A:161-168.

Cools AM, Dewitte V, Lanszweert F, Notebaert D, Roets A, Soetens B, Cagnie B, Witvrouw EE. Rehabilitation of scapular muscle balance: which exercises to prescribe? Am J Sports Med 2007;35:1744-1751.

Cools AM, Struyf F, De Mey K, Maenhout A, Castelein B, Cagnie B. Rehabilitation of scapular dyskinesis: from the office worker to the elite overhead athlete. Br J Sports Med 2014;48:692-697.

Eklund D, Pulverenti T, Bankers S, Avela J, Newton R, Schumann M, Häkkinen K. Neuromuscular adaptations to different modes of combined strength and endurance training. Int J Sports Med 2015;36:120-129.

Fatemi R, Javid M, Najafabadi EM. Effects of William training on lumbosacral muscles function, lumbar curve and pain. J Back Musculoskelet Rehabil 2015;28:591-597.

Fathollahnejad K, Letafatkar A, Hadadnezhad M. The effect of manual therapy and stabilizing exercises on forward head and rounded shoulder postures: a six-week intervention with a one-month follow-up study. BMC Musculoskelet Disord 2019;20:86.

Feng Q, Wang M, Zhang Y, Zhou Y. The effect of a corrective functional exercise program on postural thoracic kyphosis in teenagers: a randomized controlled trial. Clin Rehabil 2018;32:48-56.

Garber CE, Blissmer B, Deschenes MR, Franklin BA, Lamonte MJ, Lee IM, Nieman DC, Swain DP; American College of Sports Medicine. American College of Sports Medicine position stand. Quantity and quality of exercise for developing and maintaining cardiorespiratory, musculoskeletal, and neuromotor fitness in apparently healthy adults: guidance for prescribing exercise. Med Sci Sports Exerc 2011;43:13341359.

González-Gálvez N, Gea-García GM, Marcos-Pardo PJ. Effects of exercise programs on kyphosis and lordosis angle: a systematic review and meta-analysis. PLoS One 2019;14:e0216180.

Harman K, Hubley-Kozey CL, Butler H. Effectiveness of an exercise program to improve forward head posture in normal adults: a randomized, controlled 10-week trial. J Man Manip Ther 2005;13:163-176.

Hamill J, van Emmerik RE, Heiderscheit BC, Li L. A dynamical systems approach to lower extremity running injuries. Clin Biomech (Bristol, Avon) 1999;14:297-308.

Higgins JP, Altman DG, Gøtzsche PC, Jüni P, Moher D, Oxman AD, Savovic J, Schulz KF, Weeks L, Sterne JA; Cochrane Bias Methods Group; Cochrane Statistical Methods Group. The Cochrane Collaboration's tool for assessing risk of bias in randomised trials. Version 2. BMJ 2011; 343:d5928. 
Hodges PW, van Dieën JH, Cholewicki J. Time to reflect on the role of motor control in low back pain. J Orthop Sports Phys Ther 2019;49: 367-369.

Hrysomallis C, Goodman C. A review of resistance exercise and posture realignment. J Strength Cond Res 2001;15:385-390.

Im B, Kim Y, Chung Y, Hwang S. Effects of scapular stabilization exercise on neck posture and muscle activation in individuals with neck pain and forward head posture. J Phys Ther Sci 2016;28:951-955.

Janda V. On the concept of postural muscles and posture in man. Aust J Physiother 1983;29:83-84.

Jang HJ, Hughes LC, Oh DW, Kim SY. Effects of corrective exercise for thoracic hyperkyphosis on posture, balance, and well-being in older women: a double-blind, group-matched design. J Geriatr Phys Ther 2019;42:E17-E27.

Kamali F, Shirazi SA, Ebrahimi S, Mirshamsi M, Ghanbari A. Comparison of manual therapy and exercise therapy for postural hyperkyphosis: a randomized clinical trial. Physiother Theory Pract 2016;32:92-97.

Kang JI, Choi HH, Jeong DK, Choi H, Moon YJ, Park JS. Effect of scapular stabilization exercise on neck alignment and muscle activity in patients with forward head posture. J Phys Ther Sci 2018;30:804-808.

Katzman WB, Vittinghoff E, Lin F, Schafer A, Long RK, Wong S, Gladin A, Fan B, Allaire B, Kado DM, Lane NE. Targeted spine strengthening exercise and posture training program to reduce hyperkyphosis in older adults: results from the study of hyperkyphosis, exercise, and function (SHEAF) randomized controlled trial. Osteoporos Int 2017; 28:2831-2841.

Kim KH, Kim SG, Hwangbo G. The effects of horse-riding simulator exercise and Kendall exercise on the forward head posture. J Phys Ther Sci 2015;27:1125-1127.

Ko KJ, Ha GC, Yook YS, Kang SJ. Effects of 12-week lumbar stabilization exercise and sling exercise on lumbosacral region angle, lumbar muscle strength, and pain scale of patients with chronic low back pain. J Phys Ther Sci 2018;30:18-22.

Lee DY, Nam CW, Sung YB, Kim K, Lee HY. Changes in rounded shoulder posture and forward head posture according to exercise methods. J Phys Ther Sci 2017;29:1824-1827.

Lee SM, Lee CH, O'Sullivan D, Jung JH, Park JJ. Clinical effectiveness of a Pilates treatment for forward head posture. J Phys Ther Sci 2016;28: 2009-2013.

Lynch SS, Thigpen CA, Mihalik JP, Prentice WE, Padua D. The effects of an exercise intervention on forward head and rounded shoulder postures in elite swimmers. Br J Sports Med 2010;44:376-381.

Magnusson SP, Simonsen EB, Aagaard P, Boesen J, Johannsen F, Kjaer M. Determinants of musculoskeletal flexibility: viscoelastic properties, cross-sectional area, EMG and stretch tolerance. Scand J Med Sci Sports

\section{7;7:195-202.}

Magnusson SP. Passive properties of human skeletal muscle during stretch maneuvers. A review. Scand J Med Sci Sports 1998;8:65-77.

McHugh ML. Interrater reliability: the kappa statistic. Biochem Med (Zagreb) 2012;22:276-282.

Morris CE, Greenman PE, Bullock MI, Basmajian JV, Kobesova A. Vladimir Janda, MD, DSc: tribute to a master of rehabilitation. Spine (Phila Pa 1976) 2006;31:1060-1064.

Muyor JM, López-Miñarro PA, Casimiro AJ. Effect of stretching program in an industrial workplace on hamstring flexibility and sagittal spinal posture of adult women workers: a randomized controlled trial. J Back Musculoskelet Rehabil 2012;25:161-169.

Pekyavas NO, Ergun N. Comparison of virtual reality exergaming and home exercise programs in patients with subacromial impingement syndrome and scapular dyskinesis: Short term effect. Acta Orthop Traumatol Turc 2017;51:238-242.

Romano M, Minozzi S, Zaina F, Saltikov JB, Chockalingam N, Kotwicki T, Hennes AM, Negrini S. Exercises for adolescent idiopathic scoliosis: a Cochrane systematic review. Spine (Phila Pa 1976) 2013;38:E883-E893.

Rubinstein SM, Terwee CB, Assendelft WJ, de Boer MR, van Tulder MW. Spinal manipulative therapy for acute low back pain: an update of the cochrane review. Spine (Phila Pa 1976) 2013;38:E158-E177.

Ruivo RM, Pezarat-Correia P, Carita AI. Effects of a resistance and stretching training program on forward head and protracted shoulder posture in adolescents. J Manipulative Physiol Ther 2017;40:1-10.

Sahrmann S, Azevedo DC, Dillen LV. Diagnosis and treatment of movement system impairment syndromes. Braz J Phys Ther 2017;21:391-399.

Schamberger W. Malalignment syndrome in runners. Phys Med Rehabil Clin N Am 2016;27:237-317.

Schwanke NL, Pohl HH, Reuter CP, Borges TS, de Souza S, Burgos MS. Differences in body posture, strength and flexibility in schoolchildren with overweight and obesity: a quasi-experimental study. Man Ther 2016;22:138-144.

Seidi F, Rajabi R, Ebrahimi I, Alizadeh MH, Minoonejad H. The efficiency of corrective exercise interventions on thoracic hyper-kyphosis angle. J Back Musculoskelet Rehabil 2014;27:7-16.

Shamseer L, Moher D, Clarke M, Ghersi D, Liberati A, Petticrew M, Shekelle P, Stewart LA; PRISMA-P Group. Preferred reporting items for systematic review and meta-analysis protocols (PRISMA-P) 2015: elaboration and explanation. BMJ 2015;350:g7647.

Sheikhhoseini R, Shahrbanian S, Sayyadi P, O'Sullivan K. Effectiveness of therapeutic exercise on forward head posture: a systematic review and meta-analysis. J Manipulative Physiol Ther 2018;41:530-539.

Thakur D, Motimath B, Raghavendra M. Forward head posture correction versus shoulder stabilization exercises effect on scapular dyskinesia 
and shoulder proprioception in athletes: an experimental study. Int J Physiother 2016;3:197-203.

van Tulder M, Furlan A, Bombardier C, Bouter L; Editorial Board of the Cochrane Collaboration Back Review Group. Updated method guidelines for systematic reviews in the cochrane collaboration back review group. Spine (Phila Pa 1976) 2003;28:1290-1299.

Vaughn DW, Brown EW. The influence of an in-home based therapeutic exercise program on thoracic kyphosis angles. J Back Musculoskelet Rehabil 2007;20:155-165. 